\title{
Economic Evaluations of Guideline-Based or Strategic Interventions for the Prevention or Treatment of Chronic Wounds
}

\author{
Marissa J. Carter
}

Published online: 11 March 2014

(C) The Author(s) 2014. This article is published with open access at Springerlink.com

\begin{abstract}
Background Costs of chronic wound care are significant, but systematic reviews of cost-effectiveness studies regarding guideline-based or strategic interventions are scarce.

Objectives Our objectives were to assess/compare the cost effectiveness of new interventions/systems designed to improve the prevention/treatment of chronic wounds in adult populations against current care and provide decision makers with information on which to base future interventions for chronic wound management.

Data Sources Data sources included PubMed, Scopus, HTA, and NHS EED.

Study Eligibility Criteria, Participants, and Interventions We included comparative health economic evaluations of interventions published in English designed to prevent or treat adult chronic wounds that were guidelinebased or strategic in nature and from which an incremental cost-effectiveness ratio or incremental net health benefit was reported or could be calculated.

Study Appraisal and Synthesis Methods Study and model characteristics and outcomes were extracted into predesigned tables. Quality assessment of studies was based
\end{abstract}

Systematic Review Registration Number: CRD42013005606 (PROSPERO).

Electronic supplementary material The online version of this article (doi:10.1007/s40258-014-0094-9) contains supplementary material, which is available to authorized users.

M. J. Carter $(\bowtie)$

Strategic Solutions, Inc., 1143 Salsbury Ave,

Cody, WY 82414, USA

e-mail: mcarter@strategic-solutions-inc.com on literature-reported methods. Studies were assigned strength of evidence ratings and recommendation level for decision makers.

Results A total of 16 health economic evaluations were included, of which ten were trial based and six were wholly model based. Only three studies had high, and five studies moderate, strength of evidence and were recommended for decision makers. All studies had some shortcomings regarding time horizon, costs, effectiveness units, and methodological reporting. Two studies had major flaws.

Limitations Limitations include missed studies published in non-English languages or not cited in searched databases; judgment bias in assessing studies.

Conclusions and Implications of Key Findings Few well conducted cost-effectiveness studies exist to guide decision makers regarding guideline-based or strategic interventions for chronic wounds.

\section{Key Points for Decision Makers}

Few good economic studies exist quantifying the cost effectiveness of strategic or guideline-based interventions in regard to chronic wound management.

The strongest evidence is for prevention and treatment of diabetic foot ulcers, prevention of pressure ulcers in long-term care settings, and treatment of patients with critical limb ischemia.

Clinical practice guidelines are proliferating in the field of chronic wound care, but large gaps exist in demonstrating their cost effectiveness. 


\section{Introduction}

Chronic wounds are those wounds that fail to heal in a timely manner or become stuck in the inflammatory phase of healing [1]. In developed countries, the incidence of chronic wounds has been growing in recent years and likened to a 'silent epidemic' [2]. The increase in the incidence of chronic wounds is due to both the aging of populations (longer lifespan) and the concurrent increase in comorbidities, such as diabetes, obesity, venous hypertension, and peripheral vascular disease [2, 3].

The most common types of chronic wounds-venous leg ulcers (VLUs), diabetic foot ulcers (DFUs), and pressure ulcers (PUs) - and their current treatments often mean that employed patients cannot work until their wounds are healed. For example, individuals with DFUs are often forced to change their nature of employment or take time off work, and can become disabled and unable to work [4]. Social contact and mobility are also frequently impacted, leading to decreased quality of life [5]. While in the last two decades advanced therapeutics have been developed as adjunct treatments, the level of evidence for their efficacy is often poor, as exemplified by a recent review of advanced VLU treatments conducted by researchers at The Johns Hopkins University Evidence-Based Practice Center [6]. Most importantly, even though many guidelines for the management of chronic wounds have been published (clinical practice guidelines [CPGs]), too many patients still do not receive the fundamental wound care and treatment described in the guidelines. According to one professional group examining the problem for VLUs (Group IV of the Pacific Vascular Symposium 6), there are too many guidelines on VLUs from too many sources; coordinated integration is difficult; and most critically, there is a need to unify evidence-based guidelines to facilitate implementation [7].

No one knows the true cost of treating chronic wounds worldwide, because country-wide surveys have not been undertaken. However, those costs are likely to be very substantial. In the UK, costs in 2005-2006 prices were estimated at £2.3-3.1 billion [8] and more recently in the USA at \$US6-15 billion [9], although, in 2010, Driver et al. [10] reported that, in the USA alone, the cost of treating DFUs was \$US39 billion. To help guide healthcare providers in the management of chronic wound care, health economics studies are available, but there are considerable limitations with many of these studies [11, 12]. Besides flaws in the health economic studies themselves (e.g. short horizon times, questionable model inputs, or incomplete or poorly structured models), in the field of wound care such studies are mostly concerned with basic wound care and advanced therapies rather than examining the costs and benefits of applying a broader strategic treatment pattern. Consequently, little thought seems to have been given to the implementation and actual testing of CPGs in practice, particularly from a cost-effectiveness point of view [13]. Because examination of CPGs and strategies to improve cost effectiveness in wound care has been poorly explored, this is the subject of this systematic review.

The objective of this systematic review, therefore, is to assess the cost effectiveness of new interventions or systems designed to improve the prevention or treatment of chronic wounds in adult populations by comparing existing systems or practices. A second objective is to provide decision makers with information on which to base future interventions for chronic wound management.

\section{Methods}

\subsection{Study Inclusion/Exclusion Criteria}

Studies were eligible for consideration if they

- Were health economic evaluations (cost-benefit, costeffectiveness, or cost-utility analyses)

- Described interventions that prevented a chronic wound from occurring or treated an existing chronic wound

- Compared guideline-based or strategic interventions with an existing practice (i.e. change of practice)

- Included at least one incremental cost-effectiveness ratio (ICER) or incremental net health benefit (INHB), or provided costs and benefits so that ICERS/INHBs could be calculated

- Used any time horizon

- Were published in English.

Studies were excluded if they were

- Cost-minimization studies

- Only described by abstracts, letters, or editorials

- Studies that described a purely product- or technically oriented intervention, such as testing of a device, drugs, dressing, or bandaging in the context of chronic wounds.

\subsection{Literature Sources}

Databases were searched from 1 January 1974 to 20 August 2013 for eligible studies in using the following text strings: ('wound' OR 'wounds' OR 'wound care' OR 'ulcer' OR 'ulcers') AND ('cost-effectiveness' OR 'cost-benefit' OR 'cost-utility' OR 'economic evaluation' OR 'economic model' OR 'decision model' OR 'decision tree'). The following databases were searched: PubMed, Scopus, Health Technology Assessment (HTA), and National Health Service Economic Evaluation Database (NHS EED). 
While the initial selection of studies was made using abstracts based on the inclusion/exclusion criteria, the final selection (and rejection of any studies) was made after reading the full text. Reviews and systematic reviews relevant to the objectives of the study were also searched for other possible studies.

\subsection{Study Classification}

Studies accepted for the systematic review were differentiated according to whether cost and effectiveness components (model or calculation inputs) were derived from a single clinical trial or study designed as part of the economic study (trial-based economic studies), or synthesized from numerous studies (modeled economic studies). If studies used a single clinical trial and modeling, they were still classified as trial-based studies. The rationale for this classification is that trial-based studies are likely to reflect a specific situation or setting but could have issues in generalization to other settings. Model-based studies under this classification are likely to reflect broader situations or settings due to more diverse model inputs, but could have larger uncertainties.

\subsection{Data Extraction}

Outcomes were extracted from included studies and transferred to pre-designed tables describing key patient and wound characteristics and ICERs/INHBs (trial-based economic studies) or model parameters and ICERs/INHBs (modeled economic studies). If ICERs/INHBs were not explicitly stated, these were calculated from the study data using the general approach of cost of strategy A-cost of strategy B/benefit of strategy A-benefit of strategy B.

\subsection{Quality Assessment of Studies}

Quality assessment of studies was based on a synthesis of the consensus health economic criteria (CHEC) list developed by Evers et al. [14] and the quality assessment tool for decision-analytic models created by Philips et al. [15] as defined in the 23-item study appraisal reported by Langer and Rogowski [16]. The only modifications made to this study appraisal were as follows. (i) The third question in the Outcomes section (directed toward trial-based economic studies) was changed to "Were outcomes appropriately incorporated into models and adequately described?" This change pertained to meta-analytical synthesis of outcomes, and none of the trial-based studies used meta-analysis. (ii) The second question in the Analysis section was scored as follows: (a) short-term horizons ( $\leq 12$ months): no discounting of costs/benefits $=1$; discounting of any costs/ benefits or not stated $=0$; (b) longer time horizons
( $>12$ months): appropriate discounting of costs and effects $=1$; partially appropriate discounting $=0.5$; no discounting or not stated $=0$. (Original scoring was as follows: (a) short time horizon ( $\leq 12$ months): not applicable; discounting of costs and effects $=1$; only costs/not motivated discount rate $=0.5$; discount rate not stated $=0$.)

Summary scores for each study were calculated (method: Table 1) but were not used per se in any subsequent weighting scheme; rather, study scores and the possible score range were developed to provide a sense of whether the checklist questions could be answered in full or partially answered in each section, whether some questions could be answered partially, or whether only few questions could be answered at all.

Each study was given a strength-of-evidence rating as used in the GRADE (Grades of Recommendation, Assessment, Development, and Evaluation) approach [17] and defined as follows: high (further research is very unlikely to change confidence in the estimate of the effect); moderate (further research is likely to have an important impact on confidence in the estimate of the effect and may change the estimate); low (further research is very likely to have an important effect on confidence in the estimate of the effect and is likely to change the estimate); and very low (any estimate of effect is very uncertain). In the context of the GRADE category definitions, effect in this systematic review means the ICER or INHB.

These ratings were assigned based on how questions were scored in each section of the quality assessment, additional strengths or weaknesses of the study that the quality assessment did not cover, and the overall strength of the underlying evidence. An example of an additional strength might be that the study employed meta-analysis from several appropriate sources to derive realistic wound healing outcomes; an example of an additional weakness might be that a model was not validated. The overall

Table 1 Scoring system used to identify strengths and weaknesses in each section of the quality assessment

\begin{tabular}{ll}
\hline Section & Permissible score \\
\hline Research question/perspective & $0-2$ \\
Interventions(s) & $0-6^{\mathrm{a}}$ \\
Costs & $0-3$ \\
Outcomes & $0-5^{\mathrm{b}}$ \\
Analysis & $0-6^{\mathrm{a}}$ \\
Total & $0-22^{\mathrm{c}}$ \\
\hline
\end{tabular}

a 5 for trial-based economic analysis and 6 for model-based economic analysis

b $0-2$ for trial-based economic analysis not using a model, and 0-5 for all others

c 17 for trial-based economic analysis (no modeling), 20 for trialbased economic analysis (modeling included), and 22 for modelbased economic analysis 
strength of the evidence is a judgment, not only about the quality of the design of the study but also the robustness and nature of the study data (input), the study results, the uncertainty surrounding those results, and therefore whether or not the conclusions are credible.

Use of each study for decision making was categorized as recommended or not recommended depending on whether the study had a high or moderate strength of evidence, and had credible and robust results for the intervention described. Recommended studies can have contextually positive or negative results-for example, the study could be given a recommendation that decision makers take note of the conclusions because the results suggest that the intervention is not likely to be cost effective.

The systematic review protocol was registered with PROSPERO (CRD42013005606) available at: http://www. crd.york.ac.uk/prospero/display_record.asp?ID=CRD4201 3005606\#.Ut6O__tlBkg.

\section{Results}

The primary search yielded 2,789 citations. A review located from the literature search, which was conducted by Franks and Bosanquet [18], yielded four additional citations, which were evaluated using full text. A total of 16 studies were included in the systematic review, with six studies rejected at the full-text assessment stage mainly due to inability to calculate an ICER or INHB (Fig. 1; Table 2).

The results of the quality assessment are shown in Online Resource 1.

\subsection{Overview}

Ten of the studies were trial-based economic studies, of which seven did not use models (Table 3) while six studies were model-based economic studies (Table 4). Strength of the evidence varied considerably for the trial-based studies, with only one study assessed as high and three as moderate; two model-based studies were assessed as having a high strength of evidence, while two others had a moderate strength of evidence.

Because the designs of the studies were so heterogeneous, meta-analysis was not possible, so each study is assessed in detail.

\subsection{Trial-Based Economic Studies (No Modeling)}

\subsubsection{Simon et al. (Strength of Evidence: Moderate; Recommended)}

The goal of the comparative before-and-after design study reported by Simon et al. [25] was to compare current leg ulcer care at one district in the UK (Stockport) in 1993 with the introduction of five leg ulcer clinics in 1994, which used nurses trained in wound care evidence-based treatments focused on vascular screening and multilayer bandaging. Three-month data-collection periods (audits) were carried out in both years, using the nearby district of Trafford as a contemporary control. The proportion of leg ulcers healed in 1994 versus 1993 (3 months) was 42 vs. $26 \%$, with healing rates at Trafford of 20 and $23 \%$, respectively. Although detailed costs were calculated and extrapolated in each year, no specific ICER was reported; by extrapolating wound healing data for 1 year, an ICER of $-£ 1,186 /$ additional healed ulcer was obtained through calculation. Principal weaknesses of the study included lack of sensitivity analysis, exclusion of more severe leg ulcers, and characteristics of the study populations, making comparison with other districts within the UK or other countries difficult. Strengths of the study included reduction of selection bias by using a geographic catchment area and the study design itself.

\subsubsection{Morrell et al. (Strength of Evidence: Moderate; Recommended)}

Morrell et al. [26] utilized a non-blinded randomized controlled trial (RCT) in which patients with VLUs were randomly assigned to community leg ulcer clinics that used four-layered bandaging or usual care provided by district nurses in the UK. Using a time horizon of 1 year and presumably a healthcare payer's perspective, results showed an ICER of $£ 2.36$ per ulcer-free week (presumed $95 \%$ confidence interval [CI] -31.94-99.12), obtained through bootstrap resampling. The authors noted poor healing rates ( $34 \%$ of wounds healed at 12 weeks in the intervention group vs. $24 \%$ in the control group), suggesting this was due to larger and older ulcers at baseline. While a factor, later results of the landmark RCT 'venUS I' [42] suggest that lack of expertise in fourlayer bandaging could have played a role. Strengths of the study were that the sample size was appropriate and the population selected fairly representative of the 'real world'. Weaknesses included some uncertainty of cost savings due to lack of transparency in data sources and reporting.

\subsubsection{Thomson et al. (Strength of Evidence: Very Low; Not Recommended)}

A pilot study conducted by Thomson and Brooks [27] focused on a projected PU prevention policy in a 252-bed geriatric unit in Glasgow (Scotland) in which current care was compared with a PU prevention policy, although details of the new program were scant. Although no ICERs 
Fig. 1 Flow chart for study selection

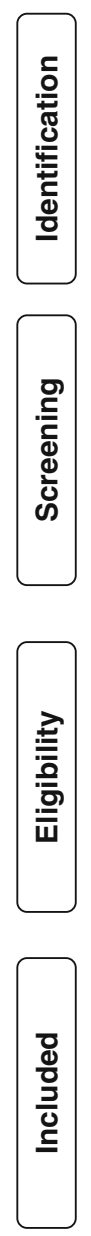

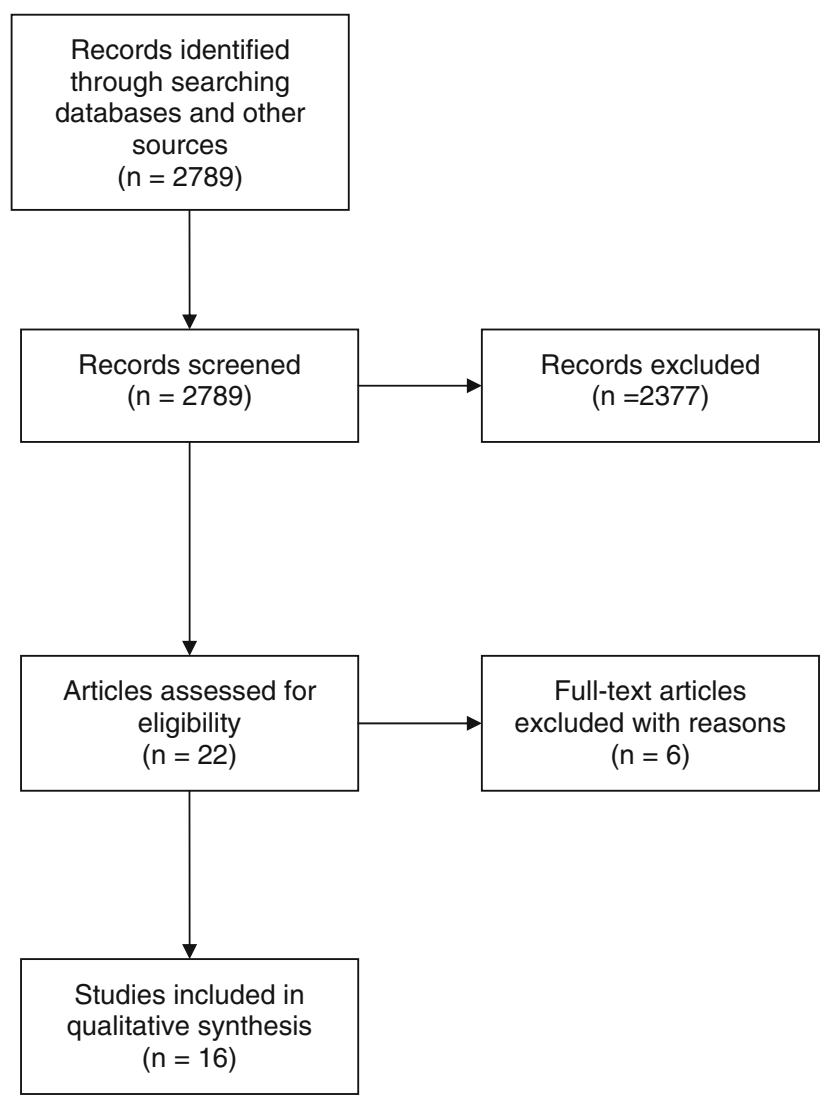

Table 2 Studies rejected for the systematic review

\begin{tabular}{ll}
\hline Study & Reason for rejection \\
\hline Bosanquet et al. [19] & Insufficient healing data to calculate INHB \\
Kerstein and Gahtan [20] & Costs/benefits not clearly indicated for the two different settings (home healthcare vs. office) \\
Kerstein et al. [21] & Study evaluated three different dressings (i.e. not strategic intervention) \\
Quioc [22] & Case study of two patients \\
Ellison et al. [23] & Insufficient data to calculate INHB \\
Schuurman et al. [24] & Cost minimization study; insufficient data to calculate INHB
\end{tabular}

$I N H B$ incremental health benefit

or INHBs were reported, calculations showed that cost savings of $£ 7,717$ would be achieved for every averted PU. Weaknesses of the study included lack of program details, no patient population description, lack of sensitivity analysis, and lack of credible data used to develop PU prevalence in the 'program' arm.

\subsubsection{Ohura et al. (Strength of Evidence: Low; Not Recommended)}

This Japanese study [28, 29] was carried out in a hospital setting to examine the cost effectiveness of three different strategies to treat stage II and III PUs: (i) modern dressings and standardized wound management, and traditional dressings (ii) with and (iii) without standardized wound management. Wound healing assessment was characterized using the Pressure Sore Status Tool (PSST) with a cohort of 83 patients over a period of 12 weeks. Based on costs and healing results, the ICER comparing strategies (i) and (iii) was $-\$ 448$ per PSST point reduction, with strategy (i) being dominant. Weaknesses of the study included the small sample size, lack of explicit perspective, the unit of benefit employed, and lack of sensitivity analysis. 


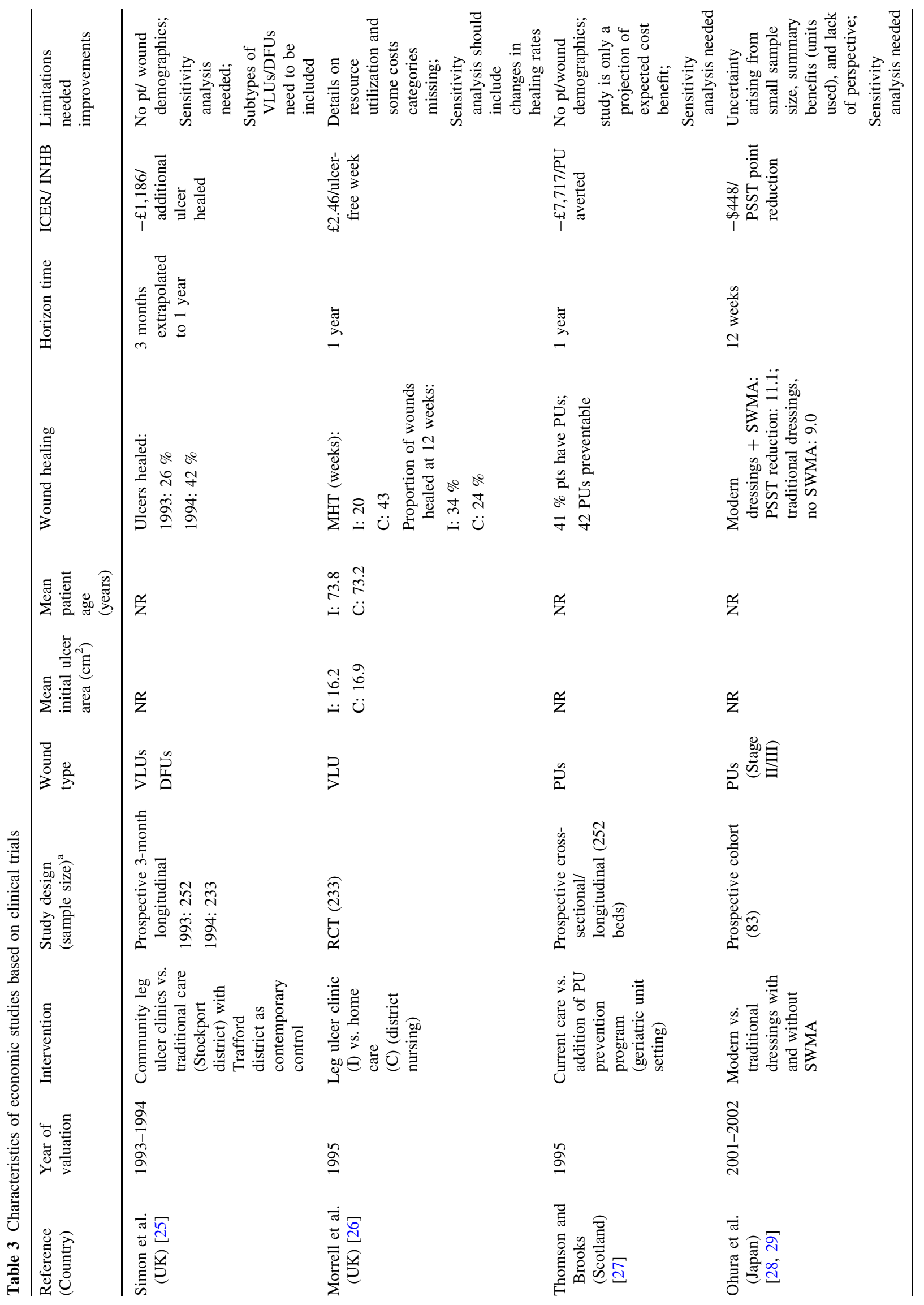




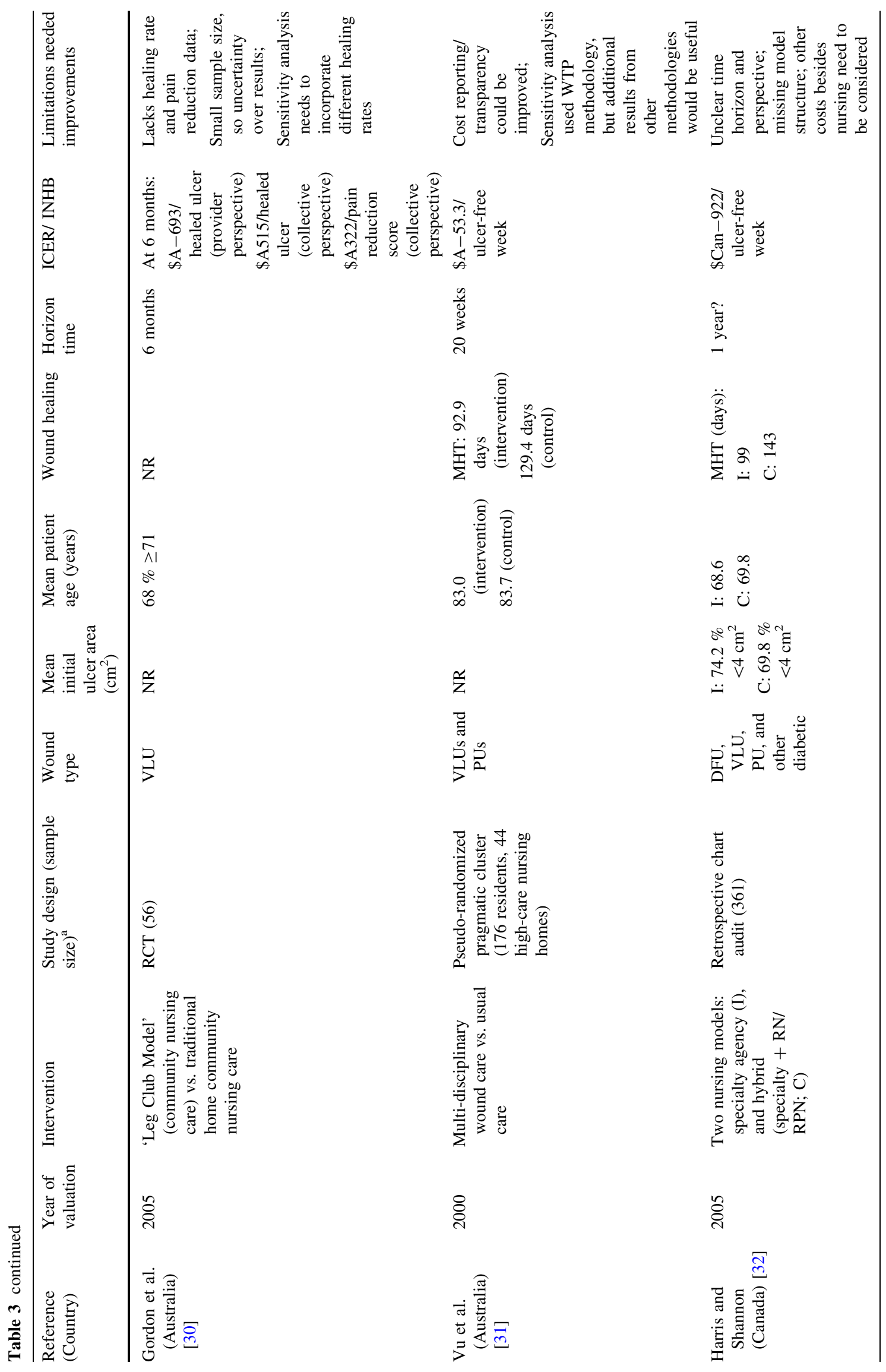




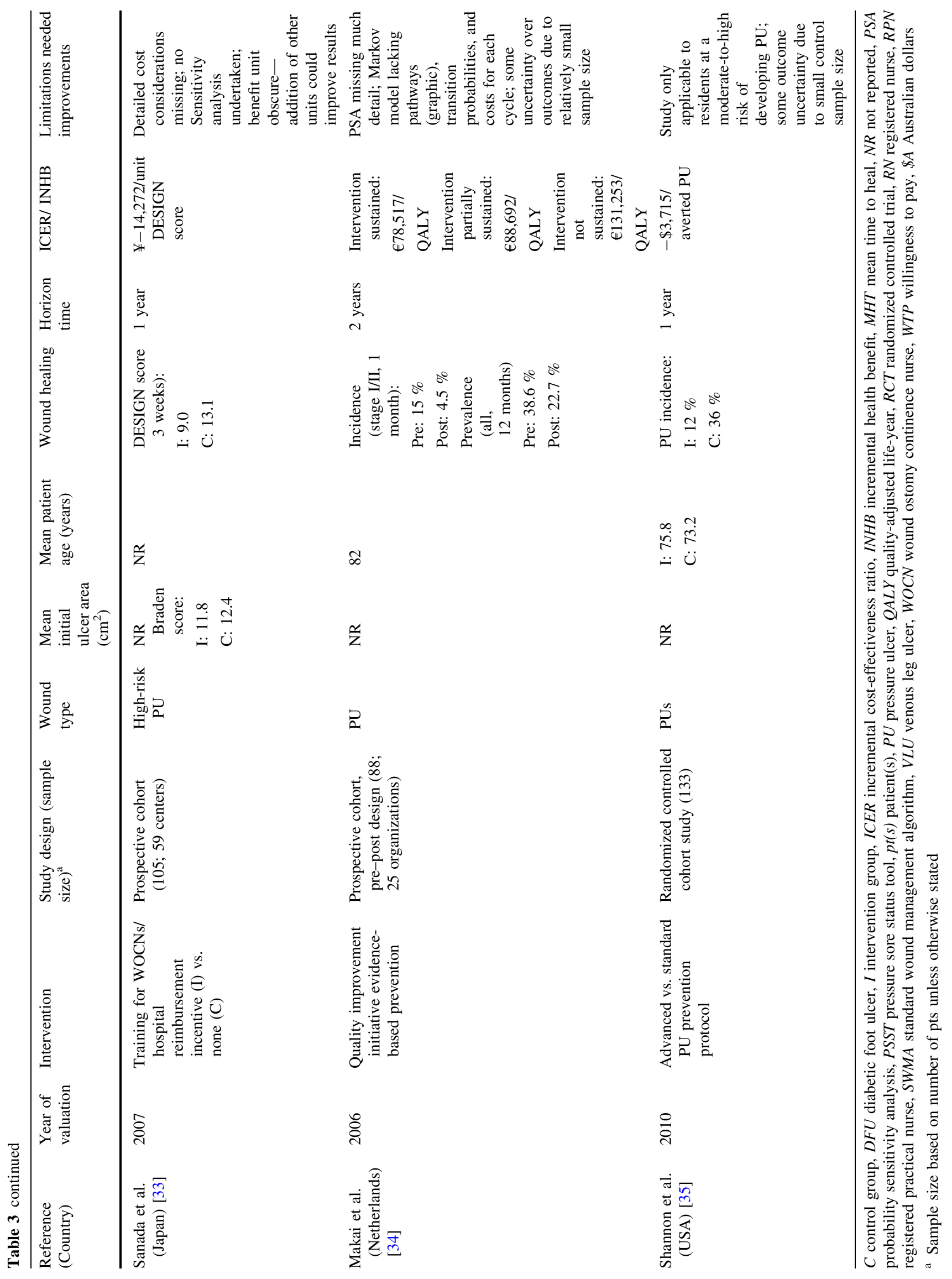




\subsubsection{Gordon et al. (Strength of Evidence: Low; Not Recommended)}

A similar albeit smaller trial to the one conducted by Morrell et al. [26] was conducted by Gordon et al. [30], in which participants with VLUs were randomized to either the Lindsay Leg Club Model or home (visit) community care in Australia; the Leg Club Model is an informal socialinteractive-promotional setting in which patients receive care similar to traditional home care once a week. From a 'collective' perspective, equivalent to a societal perspective, at 6 months the incremental cost per healed ulcer was (in Australian dollars [\$A]) \$A515 and \$A322 per reduced pain score, reflecting improved healing rates and reduced pain scores in the Leg Club patients. From a healthcare provider perspective, costs were always less and patients had higher healing rates at 3 and 6 months for the Leg Club group than for the control group. Weaknesses of the study included the short time horizon, lack of healing and pain data, small sample size, awkward benefit units, and no consideration of healing rate changes in the sensitivity analysis. A strength of the study was that some community and patient costs were assessed.

\subsubsection{Vu et al. (Strength of Evidence: Moderate; Recommended)}

$\mathrm{Vu}$ et al. [31] conducted an unusual pseudo-randomized pragmatic cluster trial of 342 VLUs and PUs in 176 elderly residents of 44 high-care nursing homes to determine whether introduction of a multidisciplinary wound care team was cost effective compared with usual care over a period of 20 weeks. Adjusted time-to-heal analysis showed that wounds healed faster in the intervention group than in the control group, by 36.5 days. Combined with cost-result data, calculations showed a dominant ICER of $\$ A-53.3$ per ulcer-free week gained. Net benefits and their $95 \%$ CIs were always positive for any non-negative societal willingness to pay (WTP) for a day without a chronic wound. Weaknesses of the study included lack of cost details, while strengths of the study included sample size, the methods used to analyze the data adjusting for clustering effects, and the WTP results. The study would also have benefitted from further sensitivity analysis.

\subsubsection{Sanada et al. (Strength of Evidence: Low; Not Recommended)}

Using a cohort design, Sanada et al. [33] in Japan evaluated the cost effectiveness of a new incentive strategy based on the structural element of the Donabedian model [43] for PU management in hospitals, which was recently introduced by the Government. The incentive reimburses about \$US45 per admission of a patient judged at high risk for a PU if the hospital meets certain facility criteria and the introduction of full-time trained wound ostomy continence nurses as part of multidisciplinary teams to manage PUs. Patients were compared in institutions in which the new incentive had been implemented versus institutions in which it had not been implemented (105 patients, 59 institutions). Outcomes were based on the Depth, Exudate, Size, Infection, Granulation, Necrosis (DESIGN) score, a tool for classifying PU severity and monitoring progression towards healing, which is used almost exclusively in Japan [44]. At 3 weeks, the cost effectiveness was $¥ 14,272$ (equivalent to \$US143 in 2013) per 1-point reduction in the DESIGN score. Weaknesses of the study included an unstated perspective; lack of detailed resource utilization, unit costs, and reference year for non-labor costs; incomplete reference to data sources; potential for missing certain material and procedure costs; no sensitivity analysis for critical variables; small sample size and associated uncertainty of having fewer than two patients per site; and the generalizability of the cost-effectiveness results, as the DESIGN score is not used outside of Japan.

\subsection{Trial-based Economic Studies (Modeling)}

\subsubsection{Harris and Shannon (Strength of Evidence: Very Low; Not Recommended)}

In a retrospective chart review of patients with a variety of wound types in Ontario, Canada, Harris and Shannon [32] conducted a cost-effectiveness study to determine if management of wounds was more cost effective when there was involvement of nurses with enterostomal (ET) or advanced wound ostomy skills (AWOS). A probabilistic decision analysis and Monte Carlo simulation was performed between the purely ET/AWOS group and a hybrid group in which patients were treated initially by an ET/ AWOS nurse followed by general visiting nurses (registered nurses and registered practical nurses), suggested that nursing visit cost is higher for the hybrid model. Comparing patients treated by ET/AWOS nurses with those treated in a hybrid model showed a dominant ICER of \$Can-922 per ulcer-free week. Moreover, there was a significant difference in healing time and reduced costs as ET/AWOS nurses became more involved with cases. Weaknesses of the study included the lack of stated perspective and time horizon, detailed reporting of resources utilized and unit costs, additional costs besides nursing, description of the decision tree, missing data (bias issues), and the nature of the data drawn retrospectively from chart reviews. 


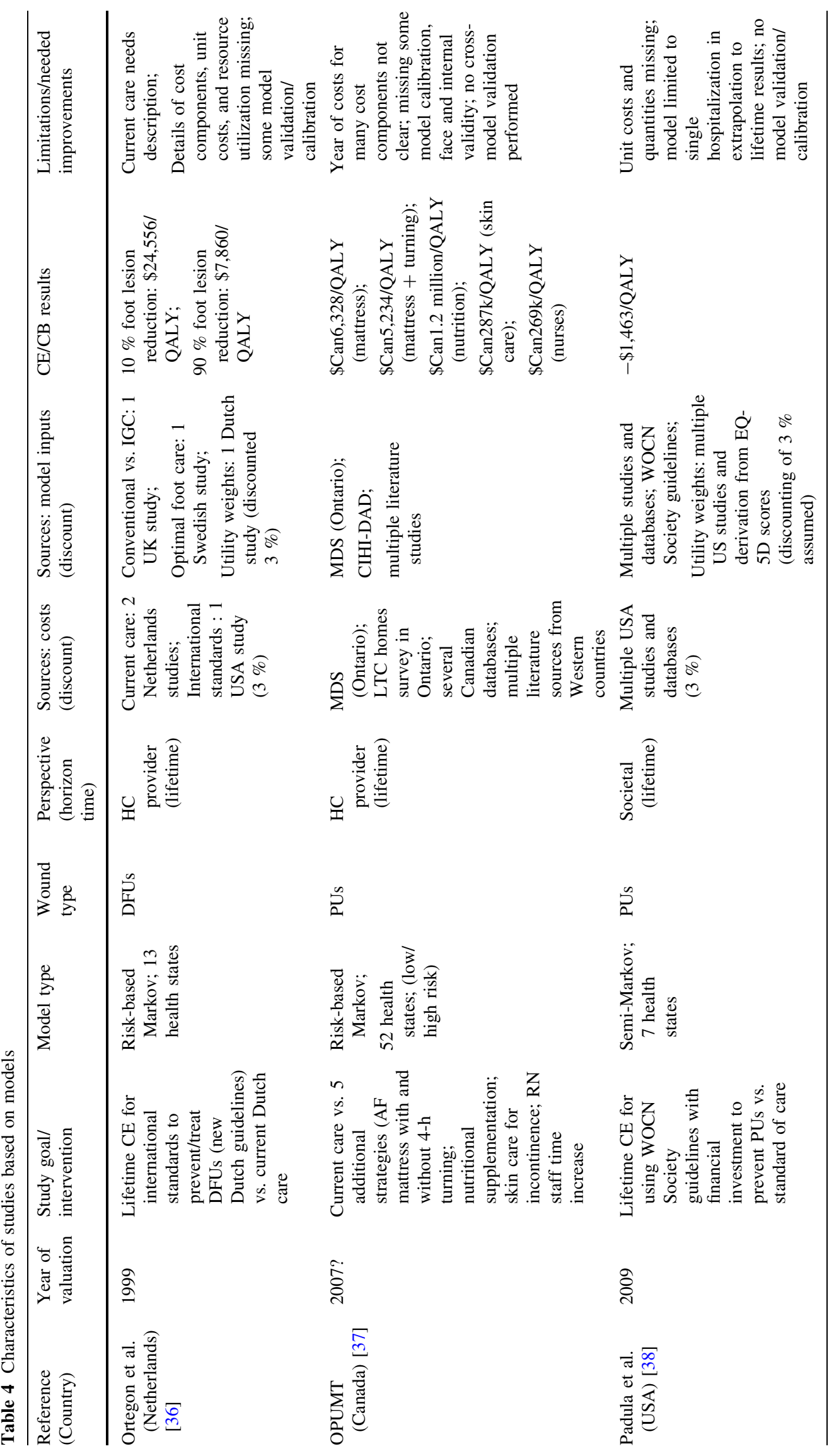




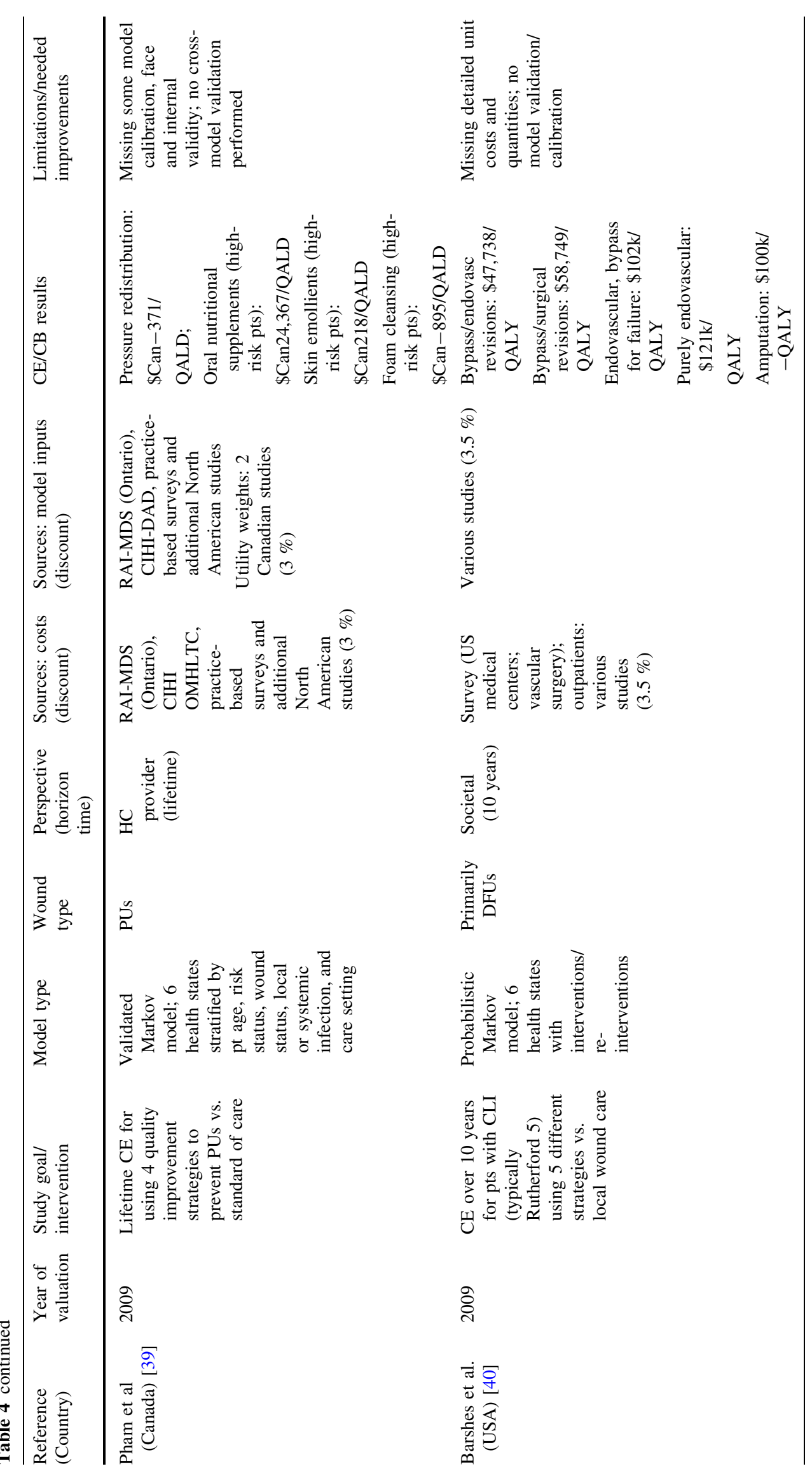




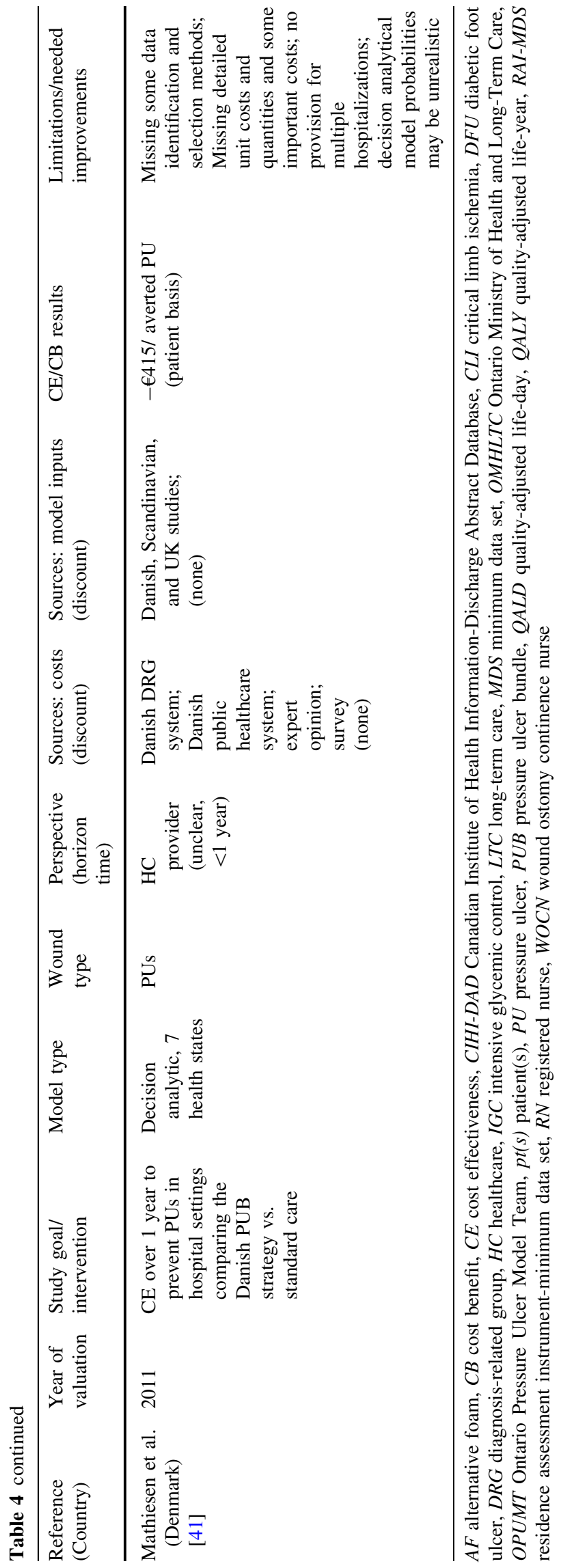

\subsubsection{Makai et al. (Strength of Evidence: Low; Not Recommended)}

Another initiative in the Netherlands concerned PUs in nursing and residential care homes in an elderly population aged 80 years or more and whose projected lifespan was short, as evidenced by the mortality rate of $30 \%$ over 1 year [34]. The prospective pre-post cohort design aimed to determine the cost effectiveness of replacing usual care with a Dutch quality improvement collaborative (QIC) in 25 organizations. The QIC has the goal of reducing the prevalence and incidence of PUs by increasing evidencebased prevention measures, including risk assessment; involvement of patients/family; use of better mattresses; preventing nutritional deficiency; and reducing non-useful measures, such as massage, $90^{\circ}$ turns every $4 \mathrm{~h}$, and use of topical agents to prevent blood supply disturbances caused by pressure. Information on prevalence, incidence, and severity of PUs was collected 1 month prior to QIC implementation, which occurred in stages. Post-QIC data were collected on 88 selected patients in round 3. The healthcare perspective was employed, with a horizon time of 2 years, modeling three scenarios projected from 1 year of data: totally sustained initiative; partially sustained; and not sustained. A Markov model including ten health states was built for an intervention arm and a standard of care group (no initiative). A probabilistic sensitivity analysis was also conducted using Monte Carlo simulations. ICERs varied from $€ 78,517$ per quality-adjusted life-year (QALY) in which the initiative was completely sustained to $€ 131,253 / \mathrm{QALY}$ where it was not sustained at all. The probability of the ICER being below $€ 80,000$ - the Dutch benchmark for cost-effective interventions on patients with high disease severity-was only $50 \%$ for the totally sustained scenario, casting doubt on whether the intervention is viable. Weaknesses of the study included lack of details for the Markov model as well as lack of validation/calibration, the rather strange probabilistic sensitivity analytical results, uncertainty over reference year for all costs, and the relatively small sample size.

\subsubsection{Shannon et al. (Strength of Evidence: High; Recommended)}

Shannon et al. [35] tested the prevention effectiveness of a new PU prevention program that followed evidence-based guidelines, standardized products, and the Minimum Data Set (MDS)-2.0 versus existing care in two nursing and rehabilitation centers. Medicare-eligible patients at risk of developing a PU were randomized to the new program or existing care and followed for a maximum of 6 months. A modified activity-based costing method tracked labor, products, and material costs. A decision analytic tree (five 
health states) was used in conjunction with a one-way sensitivity analysis to evaluate costs and benefits. Although ICERs or INHBs were not stated by the authors (results focused on cost savings), calculations showed that a cost savings of $\$ \mathrm{US} 3,715$ could be expected for each averted PU. The only weaknesses of the study were missing unit costs and the fact that the study is only applicable to residents at moderate or high risk of developing a PU. Strengths of the study include the study design itself as well as appropriate statistical power; and the credibility of the decision analytic model and the data sources used and the cost calculation methods.

\subsection{Model-Based Economic Studies}

\subsubsection{Ortegon et al. (Strength of Evidence: Moderate; Recommended)}

Ortegon et al. [36] developed a risk-based Markov model with 13 health states to answer the question of whether adopting international standards to prevent and treat DFUs versus current Dutch care was cost effective over a patient's lifetime. The perspective was that of the healthcare provider. The new strategy included intensive glycemic control (IGC) and optimal foot care based on outcome data drawn from key UK and Swedish studies, as well as others. Model results were robust to changes in costs and utility weights but sensitive to the amount of optimal foot care employed concurrent with IGC. For example, with $10 \%$ foot lesion reduction, the ICER was \$US24,556/ QALY (year 1997 values) but only \$US7,860/QALY when $90 \%$ of foot lesion reduction was accomplished. IGC by itself had an ICER of \$US32,057/QALY. For most scenarios, the authors concluded that the intervention was cost effective, with reductions in DFUs and lower extremity amputations. Weaknesses of the study included no description of current Dutch care; lack of detailed reporting regarding cost components, unit costs, and resource utilization; and lack of model calibration, face and internal model validity, and cross-model validity. Strengths of the study included meticulous model structure, assumptions, model inputs, and external validation of the model; and the exceptional care taken to model the Dutch population.

\subsubsection{OPUMT (Strength of Evidence: High; Recommended)}

The Ontario Pressure Ulcer Model Team (OPUMT) was given the task of evaluating implementation of PU prevention strategies in addition to existing care in Ontario long-term care facilities [37]. Five additional strategies were analyzed: (i) alternate foam mattresses to replace standard mattresses; (ii) adding 4-hourly turning/ repositioning for residents at high risk of developing PUs and not currently on such a schedule in addition to alternating foam mattresses; (iii) nutritional supplementation; (iv) skin care protocol for incontinence; and (v) slightly more than doubling registered nurse time per day for residents at high risk of developing PUs. A risk-based Markov model (high/low risk) with 52 health states was employed with 1-week cycles for elderly residents with a mean life expectancy of 3 years after admission; hospitalization or death from PU-related or non-PU-related causes were included. Data from several large Canadian databases and multiple studies from the literature were used as model inputs, the most important of which were also subject to probabilistic sensitivity analysis. Strategies (i) and (ii) were reasonably cost effective (\$Can6,328 and \$Can5,234/ QALY), but all other strategies were not, with ICERs $>\$$ Can250,000/QALY. The only weaknesses of the study were statement of year for some component costs, and some calibration and internal validity model details; no model cross-validation was performed. Strengths of the study included meticulous selection/calculation of model inputs from many sources, including large relevant provincial databases, a detailed and appropriate model, and appropriate resource utilization.

\subsubsection{Padula et al. (Strength of Evidence: Low; Not Recommended)}

In the USA, Padula et al. [38] modeled a guideline-based PU prevention strategy versus standard of care for 1 year for patients admitted to an acute care hospital with cost effectiveness extrapolation to the remaining lifespan of patients. A semi-Markov model utilizing seven health states was compiled using the societal perspective. In the intervention arm it was assumed that all patients experienced strict adherence to WOCN guidelines with financial investment, although details of these guidelines and what constitutes standard of care were absent. A dominant result of \$US-1,463/QALY was found in favor of the intervention arm. A major weakness of the study was extrapolation of the model to lifetime results without additional hospitalization episodes. Other weaknesses included missing unit costs and quantities and lack of model calibration/ validation.

\subsubsection{Pham et al. (Strength of Evidence: High; Recommended)}

Pham et al. [39] were also interested in preventing PUs but in the setting of long-term care facilities in which the mean age of the patients was 83 years with a life expectancy of 2.8 years. Current prevention practice was compared against four strategies: (i) pressure redistribution, (ii) oral 
nutritional supplements, (iii) skin emollients, and (iv) foam cleaning, with (ii) through (iv) applied to residents at high risk for PU development. The perspective was that of a healthcare provider. Using a Markov model built by OPUMT [37], with patients stratified by age, risk status, wound status, local or systemic infection, and care setting, they modeled each strategy against current practice. Because the increase in QALYs was minimal, the authors reported results in quality-adjusted life-days (QALDs); (i) \$Can-371/QALD; (ii) \$Can24,367/QALD; (iii) \$Can218/QALD; and (iv) \$Can-895/QALD. Strategies (i) and (iv) were determined to be cost effective. Weaknesses of the study included some calibration and internal validity model details; no model cross-validation was performed. Strengths of the study included good selection/ calculation of model inputs from many sources, a detailed and appropriate model, and appropriate resource utilization.

\subsubsection{Barshes et al. (Strength of Evidence: Moderate; Recommended)}

Barshes et al. [40] embarked on calculating cost effectiveness for patients with critical limb ischemia (CLI; typically Rutherford 5; the majority of patients would have DFUs) over 10 years from a societal perspective based on five different strategies to treat the CLI. The strategies were (i) local wound care (major amputation as indicated); (ii) primary (major amputation); (iii) bypass with up to five surgical revisions or amputation as needed/indicated; (iv) bypass with up to five endovascular interventions or amputation as needed/indicated; and (v) endovascular intervention with bypass for failure and up to four additional bypass revisions or amputation as needed/indicated. A probabilistic Markov model was developed, with 19 health states to evaluate strategies. When compared with strategy (i), the most cost-effective interventions were (iv) at \$US47,738/QALY followed by (iii) at \$US58,749/ QALY. Strategy (ii) — primary amputation-was dominated, costing over \$US100,000 for every negative QALY, meaning negative benefits and higher costs. Weaknesses of the study included the lack of the hypothetical population characteristics; absence of detailed unit costs and quantities; and no model calibration or validation details. A particular strength of the study was the overall model structure used to evaluate the five strategies.

\subsubsection{Mathiesen et al. (Strength of Evidence: Low; Not Recommended)}

The last model-based study in this systematic review was conducted by Mathiesen et al. [41] and addressed the prevention of hospital-acquired pressure ulcers (HAPUs) in Denmark. This cost-effectiveness study had a time horizon of 1 year and the perspective of a healthcare provider. It compared the Danish pressure ulcer bundle (PUB), which comprises risk assessment, daily assessment for patients at risk of developing a PU, nutrition assessment for at-risk patients, and optimal mobilization with decompression following repositioning for at-risk patients with current standard of care. A decision analytic model with seven health states was utilized to analyze cost effectiveness. While explicit ICER values were only available in graphic form, calculations indicate an ICER of $€ 415$ per PU averted. Weaknesses of the study included lack of separate reporting for resource quantities and units; missing cost categories (only labor costs were calculated); the short horizon time and no provision for multiple hospitalizations.

\section{Discussion}

\subsection{Methodological Issues}

\subsubsection{Model Structure and Assumptions}

Model structure, and the rationale/assumptions used to create it are critical for any study that employs a model to evaluate interventions or strategies. Although it can be difficult to simulate real-life pathologies, the model should be detailed enough to capture all major sequiturs likely to have an impact and discuss reasons why any relevant pathology-related health states are not included [45]. While the majority of the studies reviewed had reasonable trees or model structure, the decision tree or Markov model employed in several studies was unclear as the graphics presented were often a summary rather than a detailed tree [32, 34, 37, 39].

Although hypothetical cohorts were used in all studies utilizing decision trees or models, it was not always clear what the demographics of such populations were. For example, in the study conducted by Barshes et al. [40], it is not known whether this model would be valid for elderly patients, where the purely endovascular approach is often preferable due to higher risk of surgical mortality [46].

\subsubsection{Model Validation and Calibration}

In the field of cardiology Haji et al. [47] noted that the majority of model-based studies lacked validation and calibration; this also appears to be true in the field of wound care. With the exception of two studies $[37,39]$ in which a serious attempt was made to report model calibration and validity, little [36] or no information [34, 38, 40] was found in other Markov-based studies. This is one 
reason why only two model-based studies were given a high strength of evidence rating.

None of the studies attempted cross-model validation. While this can be construed as a weakness, in wound care there may be an insufficient number of good models from which to choose that encompass the particular pathologies, settings, and details needed to make a reasonable match.

\subsubsection{Credibility of Data Sources}

In wound care, having several robust data sources for outcomes is a common issue. One particular problem is that using data from RCTs may be too biased because such data do not reflect 'real world' situations [48]. The converse dilemma is that there is a dearth of good observational studies that are appropriately powered and for which outcomes have been adjusted for confounding variables, a recommendation endorsed by the International Society for Pharmacoeconomics and Outcomes Research (ISPOR) [45]. A few studies, including trial-based studies, did not report outcomes adequately, did not identify sources properly, or did not specify how outcomes were derived for model inputs [25, 31, 41].

Several studies [25-29, 31-33, 36, 44] did not report a detailed breakdown of costs, the reference year for pricing, or unit costs separately from quantities of resources used, all of which are needed to assess adequacy of cost compilation [49]. Moreover, some omitted relevant items could have impacted reported results [26, 32, 33, 36, 41].

\subsubsection{Indirect Costs}

Only three studies attempted to capture indirect costs by using a societal perspective [30,38,40], but in only one study [40] were such costs likely to be representative. Although the health provider cost perspective is a logical choice for most studies, because the decision maker is often the healthcare provider, such a perspective cannot inform whether a particular intervention benefits society as a whole, a detraction in the opinion of some health economists [50].

\subsubsection{Time Horizon}

All the trial-based studies had a time horizon of 1 year or less, with the exception of the study by Makai et al. [34], which was 2 years. Conversely, most of the model-based studies had time horizons of the cohorts' expected lifespan, which varied considerably; one study [41] had a time horizon of less than 1 year, albeit unclear, and one was 10 years [40].

While modeling longer time horizons can be challenging because of sparse or inaccurate data, with appropriate sensitivity analysis, uncertainties in results can be quantified to test assumptions. Studies carried out with short horizon times, on the other hand, can produce misleading results because we do not know the long-term effect of interventions in the framework of cost effectiveness [51]. The conclusions of such studies should be regarded with circumspection. Modeling longer horizon times also requires realistic data for different time periods and for different types of patients, which may not be available. Merely extrapolating a year's worth of modeled data can be misleading as exemplified by the study by Padula et al. [38], in which repeat hospitalizations were omitted, thereby creating much unquantifiable uncertainty over the results. The premise of the author's argument was to extrapolate the benefits of a better strategy for prevention and treatment of PUs incurred during a single hospitalization episode that was modeled for 1 year over the remaining patients' lifespan. Based on a mean patient age of 56 years, this is unrealistic as many patients would be likely to undergo further hospitalization episodes. A better approach would have been an undiscounted utility reward accumulated over the 1 year or provision for a hospitalization readmission pathway in the model structure, with the final utility reward being accumulated over the patients' estimated lifespan.

\subsubsection{Units of Effectiveness}

Five of the model-based studies and one trial-based study used QALYs or QALDs [34, 36-40]. Although this unit is not an ideal effectiveness unit [52] and requires assessment of utilities, which for some chronic wounds can be a challenge, it does enable cost effectiveness of studies to be compared against each other. The remaining studies either used ulcer-free weeks or months (or calculations were made in these units) or progressively more obscure effectiveness units, which meant that most of the ICERs or INHBs could not easily be related to any other health economic studies in wound care nor easily assessed against any common cost-effectiveness benchmarks.

\subsection{Strengths and Limitations of the Present Study}

A major strength of this systematic review is that included studies were subject to rigorous quality review, and the assessment paralleled the GRADE approach where feasible. A second strength of this review is that it followed Preferred Reporting Items for Systematic Reviews and Meta-Analyses (PRISMA) guidelines, although some guidelines could not be applied due to the nature of the study. In terms of limitations, it is possible that some economic studies might have been missed because they were published in languages other than English or were not 
cited in the searched databases. Second, studies are subject to some judgment bias, even when using a comprehensive appraisal system and it is possible that some studies might be evaluated slightly differently.

\subsection{Conclusions}

Few well conducted cost-effectiveness studies exist that can be used to guide decision makers in the field of chronic wounds when the intervention is strategic in nature or is based on guidelines. Although some evidence exists that it is more cost effective to treat VLUs in specialized (wound care) clinics, elements of organization and training need to be better analyzed in the cost-effectiveness context. For diabetic patients, one well executed model-based study demonstrated that intensive glycemic control in conjunction with optimal foot care was cost effective over patients' remaining lifespan; the strength of the evidence was moderate. There is also good evidence that certain interventions are cost effective as part of a strategic approach to prevent PUs in long-term care settings; however, in acutecare facilities, similar approaches have yet to demonstrate cost effectiveness. Finally, one other study demonstrated clear cost-effective differences in approaches to treating patients with CLI, but patient characteristics must also be taken into consideration.

Study Funding and Competing Interests The author declares that this study was not funded and that she has no competing interests or financial conflicts of interest.

Open Access This article is distributed under the terms of the Creative Commons Attribution Noncommercial License which permits any noncommercial use, distribution, and reproduction in any medium, provided the original author(s) and the source are credited.

\section{References}

1. Werdin F, Tennenhaus M, Schaller HE, Rennekampff HO. Evidence-based management strategies for treatment of chronic wounds. Eplasty. 2009;9:e19.

2. Sen CK, Gordillo GM, Roy S, Kirsner R, Lambert L, Hunt TK, et al. Human skin wounds: a major and snowballing threat to public health and the economy. Wound Repair Regen. 2009;17: 763-71.

3. Gist S, Tio-Matos I, Falzgraf S, Cameron S, Beebe M. Wound care in the geriatric client. Clin Interv Aging. 2009;4:269-87.

4. Waters N, Holloway S. Personal perceptions of the impact of diabetic foot disease on employment. Diabetic Foot Canada. 2013;1:32-40.

5. Price P, Krasner DL. Health-related quality of life and chronic wounds: evidence and implications for practice. In: Krasner DL, Rodeheaver GT, Sibbald RG, Woo KY, editors. Chronic wound care, a clinical source book for healthcare professionals. 5th ed. Malvern: HMP Communications; 2012. p. 77-84.
6. Lazarus G, Valle MF, Malas M, Qazi U, Maruthur NM, Doggett $\mathrm{D}$, et al. Chronic venous leg ulcer treatment: future research needs. Wound Repair Regen. 2014;22:34-42.

7. Passman MA. Non-medical initiatives to decrease venous ulcers prevalence. J Vasc Surg. 2010;52:29S-36S.

8. Posnett J, Franks PJ. The burden of chronic wounds in the UK. Nurs Times. 2008;104:44-5.

9. Markova A, Mostow EN. US skin disease assessment: ulcer and wound care. Dermatol Clin. 2012;30:107-11.

10. Driver VR, Fabbi M, Lavery LA, Gibbons G. The costs of diabetic foot: the economic case for the limb salvage team. J Am Podiatr Med Assoc. 2010;100:335-41.

11. Carter MJ. Cost-effectiveness research in wound care: definitions, approaches, and limitations. Ostomy Wound Manag. 2010;56: 48-59.

12. Carter MJ. Health economics information in wound care: the elephant in the room. Adv Wound Care. 2013;2:563-70.

13. Warriner RA 3rd, Carter MJ. The current state of evidence-based protocols in wound care. Plast Reconstr Surg. 2011;127(Suppl 1): 144 S-53S.

14. Evers S, Goossens M, Vet H, van Tulder M, Ament A. Criteria list for assessment of methodological quality of economic evaluations: consensus on health economic criteria. Int $\mathbf{J}$ Technol Assess Health Care. 2005;21:240-5.

15. Philips Z, Ginnelly L, Sculpher M, Claxton K, Golder S, Riemsma $\mathrm{R}$, et al. A review of guidelines for good practice in decision-analytic modelling in health technology assessment. Health Technol Assess 2004;8:iii-iv, ix-xi-1-158.

16. Langer A, Rogowski W. Systematic review of economic evaluations of human cell-derived wound care products for the treatment of venous leg and diabetic foot ulcers. BMC Health Serv Res. 2009;9:115.

17. Atkins D, Best D, Briss PA, Eccles M, Falck-Ytter Y, Flottorp S, et al. Grading quality of evidence and strength of recommendations. BMJ. 2004;328:1490.

18. Franks PJ, Bosanquet N. Cost-effectiveness: seeking value for money in lower extremity wound management. Int J Low Extrem Wounds. 2004;3:87-95.

19. Bosanquet N, Franks P, Moffatt C, Connolly M, Oldroyd M, Brown P, et al. Community leg ulcer clinics: cost-effectiveness. Health Trends. 1993;25:146-8.

20. Kerstein MD, Gahtan V. Outcomes of venous ulcer care: results of a longitudinal study. Ostomy Wound Manag. 2000;46:22-9.

21. Kerstein MD, Gemmen E, van Rijswijk L, Lyder CH, Phillips T, Xakellis G, et al. Cost and cost effectiveness of venous and pressure ulcer protocols of care. Dis Manag Health Outcomes. 2001;9:651-63.

22. Quioc V. Cost effectiveness in leg ulcer management in the community. Br J Community Nurs. 2001;6:276-82.

23. Ellison DA, Hayes L, Lane C, Tracey A, McCollum CN. Evaluating the cost and efficacy of leg ulcer care provided in two large UK health authorities. J Wound Care. 2002;11:47-51.

24. Schuurman JP, Schoonhoven L, Defloor T, van Engelshoven I, van Ramshorst B, Buskens E. Economic evaluation of pressure ulcer care: a cost minimization analysis of preventive strategies. Nurs Econ 2009;27(6):390-400, 415.

25. Simon DA, Freak L, Kinsella A, Walsh J, Lane C, Groarke L, et al. Community leg ulcer clinics: a comparative study in two health authorities. BMJ. 1996;312:1648-51.

26. Morrell CJ, Walters SJ, Dixon S, Collins KA, Brereton LM, Peters J, et al. Cost effectiveness of community leg ulcer clinics: randomised controlled trial. BMJ. 1998;316:1487-91.

27. Thomson JS, Brooks RG. The economics of preventing and treating pressure ulcers: a pilot study. J Wound Care. 1999;8: $312-6$. 
28. Ohura T, Sanada H, Mino Y. Clinical activity-based cost effectiveness of traditional versus modern wound management in patients with pressure ulcers [Translation]. Wounds. 2004;16: 157-63.

29. Ohura T, Sanada H, Mino Y. Clinical activity-based cost effectiveness of traditional versus modern wound management in patients with pressure ulcers. Article in Japanese. Nihon Ronen Igakkai Zasshi. 2004;41:82-91.

30. Gordon L, Edwards H, Courtney M, Finlayson K, Shuter P, Lindsay E. A cost-effectiveness analysis of two community models of care for patients with venous leg ulcers. J Wound Care. 2006;15:348-53.

31. Vu T, Harris A, Duncan G, Sussman G. Cost-effectiveness of multidisciplinary wound care in nursing homes: a pseudo-randomized pragmatic cluster trial. Fam Pract. 2007;24:372-9.

32. Harris C, Shannon R. An innovative enterostomal therapy nurse model of community wound care delivery. J Wound Ostomy Continence Nurs. 2008;35:169-83.

33. Sanada H, Nakagami G, Mizokami Y, Minami Y, Yamamoto A, Oe M, et al. Evaluating the effect of the new incentive system for high-risk pressure ulcer patients on wound healing and costeffectiveness: a cohort study. Int J Nurs Stud. 2010;47:279-86.

34. Makai P, Koopmanschap M, Bal R, Nieboer AP. Cost-effectiveness of a pressure ulcer quality collaborative. Cost Eff Resour Alloc. 2010;8:11.

35. Shannon RJ, Brown L, Chakravarthy D. Pressure ulcer prevention program study: a randomized, controlled prospective comparative value evaluation of 2 pressure ulcer prevention strategies in nursing and rehabilitation centers. Adv Skin Wound Care. 2012;25:450-64.

36. Ortegon MM, Redekop WK, Niessen LW. Cost-effectiveness of prevention and treatment of the diabetic foot. Diabetes Care. 2004;27:901-7.

37. Ontario Pressure Ulcer Model Team (OPUMT). The cost-effectiveness of prevention strategies for pressure ulcers in long-term care homes in Ontario: Projections of the Ontario Pressure Ulcer Model. THETA Report \#001, 2008. Retrieved January 12, 2014 from: http://lgdata.s3-website-us-east-1.amazonaws.com/docs/37 44/928426/TR_2008.1_theta_pressure_ulcer_prevention_in_ltc_ report.pdf.

38. Padula WV, Mishra MK, Makic MB, Sullivan PW. Improving the quality of pressure ulcer care with prevention: a cost-effectiveness analysis. Med Care. 2011;49:385-92.
39. Pham B, Stern A, Chen W, Sander B, John-Bapiste A, Thein HH, et al. Preventing pressure ulcers in long-term care: a cost-effectiveness analysis. Arch Intern Med. 2011;171:1839-47.

40. Barshes NR, Chambers JD, Cohen J, Belkin M. Cost-effectiveness in the contemporary management of critical limb ischemia with tissue loss. J Vasc Surg. 2012;56(1015-24):e1.

41. Mathiesen AS, Nørgaard K, Andersen MF, Møller KM, Ehlers LH. Are labour-intensive efforts to prevent pressure ulcers costeffective? J Med Econ. 2013;16:1238-45.

42. Iglesias C, Nelson EA, Cullum NA, Torgerson DJ. VenUS I: a randomised controlled trial of two types of bandage for treating venous leg ulcers. Health Technol Assess 2004;8:iii, 1-105.

43. Donabedian A. The quality of medical care. Science. 1978;200: 856-64.

44. Sanada H, Moriguchi T, Miyachi Y, Ohura T, Nakajo T, Tokunaga $\mathrm{K}$, et al. Reliability and validity of DESIGN, a tool that classifies pressure ulcer severity and monitors healing. J Wound Care. 2004;13:13-8.

45. Caro JJ, Briggs AH, Siebert U, Kuntz KM. Modeling good research practices-overview: a report of the ISPOR-SMDM Modeling Good Research Practices Task Force-1. Value Health. 2012;15:796-803.

46. Arvela E, Venermo M, Söderström M, Korhonen M, Halmesmäki $\mathrm{K}$, Albäck $\mathrm{A}$, et al. Infrainguinal percutaneous transluminal angioplasty or bypass surgery in patients aged 80 years and older with critical leg ischaemia. Br J Surg. 2011;98:518-26.

47. Haji Ali Afzali H, Gray J, Karnon J. Model performance evaluation (validation and calibration) in model-based studies of therapeutic interventions for cardiovascular diseases : a review and suggested reporting framework. Appl Health Econ Health Policy. 2013;11:85-93.

48. Serena T, Bates-Jensen B, Carter MJ, Cordrey R, Driver V, Fife $\mathrm{CE}$, et al. Consensus principles for wound care research obtained using a Delphi process. Wound Repair Regen. 2012;20:284-93.

49. Siegel JE, Weinstein MC, Russell LB, Gold MR. Recommendations for reporting cost-effectiveness analyses. JAMA. 1996;276:1339-41.

50. Johannesson M, O'Conor RM. Cost-utility analysis from a societal perspective. Health Policy. 1997;39:241-53.

51. Weinstein MC, Siegel JE, Gold MR, Kamlet MS, Russell LB. Recommendation of the panel on cost-effectiveness in health and medicine. JAMA. 1996;276:1253-8.

52. Neumann PJ, Greenberg D. Is the United States ready for QALYS? Health Aff (Millwood). 2009;28:1366-71. 